\title{
Giant Bladder Stones Presentation and Management
}

\author{
Alaa Eddin Obeid, Ahmed Alrashed, Hussain Alaradi, Ali Alaradi, Abdolsalam Ahmadi, Akbar Jalal \\ Department of Urology, Salmaniya Medical Complex, Road 2904, Manama 329, Kingdom of Bahrain.
}

\begin{abstract}
Corresponding Author:
Dr Alaa Eddin Obeid

Email: aeo10501@rcsi-mub.com

This is an Open Access article distributed under the terms of the Creative Commons Attribution License (creativecommons.org/ licenses/by/3.0).
\end{abstract}

Received

Accepted

Published

October 26, 2020

January 11,2021

February 5, 2021

\begin{abstract}
Background: Urolithiasis is third most frequent urological disease. The incidence of giant bladder stones is rare and there is minimal data on the causes and management of such presentation. Case Report: We hereby report a 41-year-old male who presented with acute urinary obstruction. X-ray KUB views of the abdomen showed a large calcified stone occluding the urinary bladder. The percutaneous cystolithotripsy (PCCL) with concurrent cystoscopy relieved his symptoms. Conclusion: In this paper we will present a case of a 41-year-old male who presented with lower urinary tract symptoms and was later found to have post-renal acute kidney injury due to two bladder stones largest measuring $4.3 \mathrm{~cm}$.
\end{abstract}

Keywords: : Acute Kidney Injury, Cystoscopy, Kidney, Urinary Bladder Neck Obstruction, Urolithiasis, Urologic Diseases.

\section{Introduction}

It is estimated that bladder stones comprise around $5 \%$ of urinary tract stones [1]. Spontaneous primary formations of bladder stones have been reported in literature, but it is largely seen in pediatric patients [2]. On the other hand, secondary bladder stones arise due to multiple causes which lead to urinary stasis and stagnation within the bladder. These are attributed to conditions affecting the bladder muscle activity such as neurogenic bladder, spinal cord injury, or medication side effects [2,3]. The common symptoms of bladder stones include urinary retention, recurrent urinary tract infections, and hematuria [4]. In this report we describe a case of a 41-year-old male patient who presented to the accident and emergency department with severe post-obstructive acute kidney injury (AKI) due to two bladder stones.

\section{Case-Report}

The patient, known case of controlled hypertension presented to the accidents and emergency department (A\&E) with supra-pubic progressive pain and inability to pass urine for 2 days duration. The patient did not report a history of any similar episode in the past. Since the symptoms began, the amount of voided urine decreased progressively until no urine output was present. The associated symptoms were mixed between irritative and voiding symptoms and included urgency, feeling incomplete void, hesitancy and dark urine color. Two months earlier he started to develop mild dysuria however the burning sensation increased few weeks prior to his presentation to the accidents and emergency (A\&E) department.

The patient reported that 8 months prior to his presentation he had the feeling of passing small stones like structures in urine. There was no history of red colored urine, clots, nausea, vomiting or other systemic symptoms. He did not have any prior surgical procedures. On physical examination, the patient was afebrile to $37^{\circ} \mathrm{C}$, heart rate 80 beats $/ \mathrm{min}$, blood pressure $126 / 80 \mathrm{mmHg}$. The abdomen was soft, lax with suprapubic tenderness on superficial palpation. The genital examination was normal. All other systems examination was unremarkable. On 
admission, the laboratory findings revealed a white blood cells (WBC) count of $13.26 \times 10^{9}$ cells $/ \mathrm{L}$ with left shift, serum creatinine of $134 \mu \mathrm{mol} / \mathrm{L}$ and blood urea of $11.1 \mathrm{mmol} / \mathrm{L}$. Urinalysis revealed the following $>100$ red blood cells (RBC)/hpf, 10-25 white blood cells (WBC)/hpf, negative leukocyte esterase and sterile bacterial urine culture. AP and lateral X-ray KUB (kidney, ureter \& bladder) views of the abdomen showed a large calcified stone [Fig.1,2] nearly occluding the urinary bladder as well as a smaller urethral stone. Renal ultrasound showed a distended urinary bladder with thickened bladder wall of $1 \mathrm{~cm}$ likely due to inflammation along with two variable sized shadowing calculi seen within the urinary bladder, the largest of which measures about $4.3 \mathrm{~cm}$ associated with bilateral hydronephrosis and slight alteration in the renal parenchymal echogenicity [Fig.3]. A Foley's catheter size 18 was inserted on admission and the urethral stone was pushed back into the bladder and the obstruction was relieved. The patient passed more than $700 \mathrm{ml}$ of very dark urine.

To manage the two bladder stones, the patient underwent percutaneous cystolithotripsy (PCCL) with concurrent cystoscopy. A cystoscope was inserted, ureter, prostate, bladder and the two stones were visualized. A suprapubic incision was made and a nephroscope was inserted under vision into the bladder. The calculus was fragmented using ultrasound lithotripsy through the suprapubic approach for them to pass through the urethral Amplatz. At the end of the procedure, a Foleys catheter was inserted. The catheter was then removed on the second post-operative day and the patient urinary output was normal. The patient was fit for discharge on the third post-operative day. A post-operative X-ray KUB was done and confirmed the resolution of the bladder stones [Fig.4]. The post-operative period was uneventful. The patient 2-week outpatient clinic (OPD) follow up visit was unremarkable and without any active complaint.

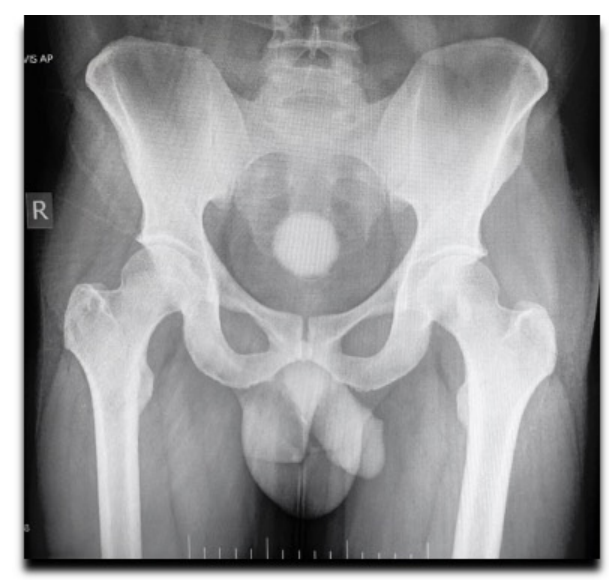

Fig.1: X-ray KUB AP view showing centrally located urinary bladder stone.

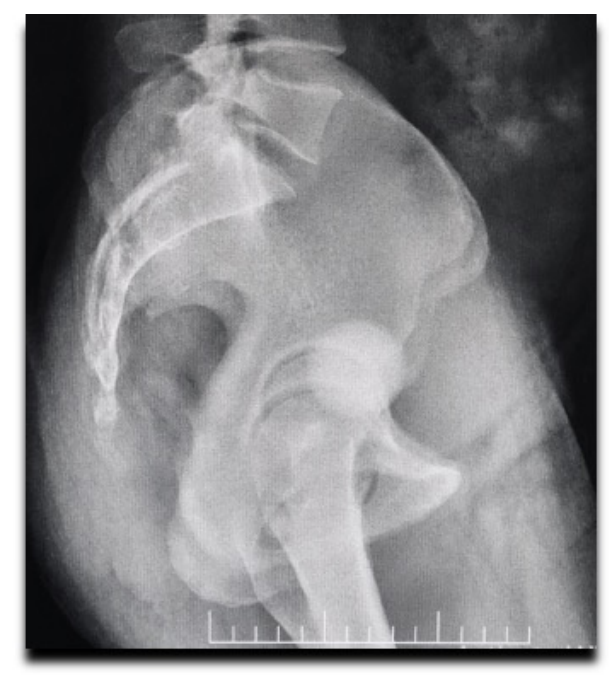

Fig.2: X-ray KUB lateral view showing large urinary stone.

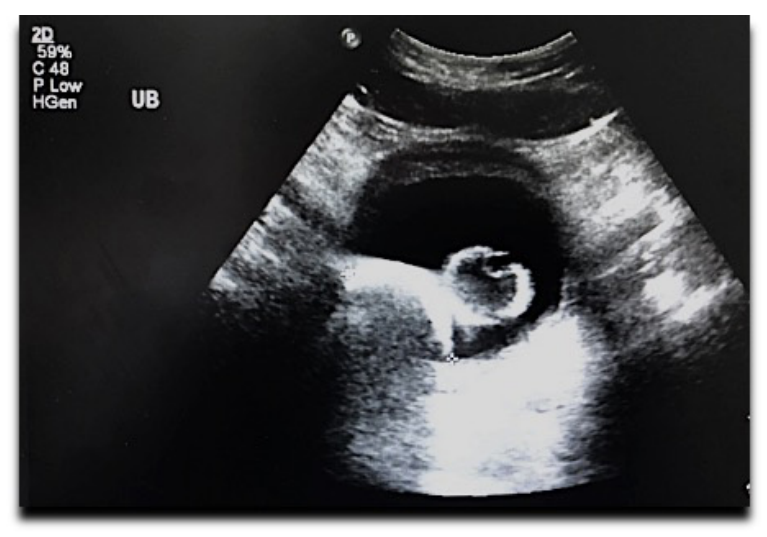

Fig.3: Bladder ultrasound (US). 


\section{Discussion}

This particular case demonstrates an uncommon presentation of large bladder stone in a patient who did not previously suffer from any chronic urinary issue. This is augmented by the fact that this is the first presentation for this patient in addition to the fact that he has no previous discernable risk factors for the formation of a bladder stones. There are different surgical interventions available for the management of large bladder stones [5]. Urologists may go for the traditional open vesicolithotomy or extracorporeal shock wave lithotripsy and transurethral pneumatic cystolithotripsy, however; clinical decision between minimally invasive surgery or open cystolithotomy depends on the number and the size of the stones $[5,6]$.

In this case the patient underwent a minimally invasive percutaneous suprapubic cystolithotripsy (PCCL). This technique is effective and safe for treating large bladder calculi, it exerts minimal trauma on the urethra and bladder [6]. In addition, it is associated with reduced hospital stay and less morbidity [6]. The patient did not have any contraindications for such technique such as a history of previous lower abdominal, pelvic surgery or bladder cancer. When compared to traditional open methods, percutaneous suprapubic cystolithotripsy showed similar stone free and recurrence rates with less post-operative pain for the patients [6]. There are limitations to our case report; a CT KUB (kidney, ureter \& bladder) is the radiographic image of choice if urolithiasis is suspected however it was not requested to the patient due to financial issues. In addition, we were not able to analyze the composition of the bladder stones due to technical issues in the laboratory equipment.

\section{Conclusion}

There is a wealth of information addressing the incidence, presentation and management for kidney and ureteral stones; nonetheless there are limited

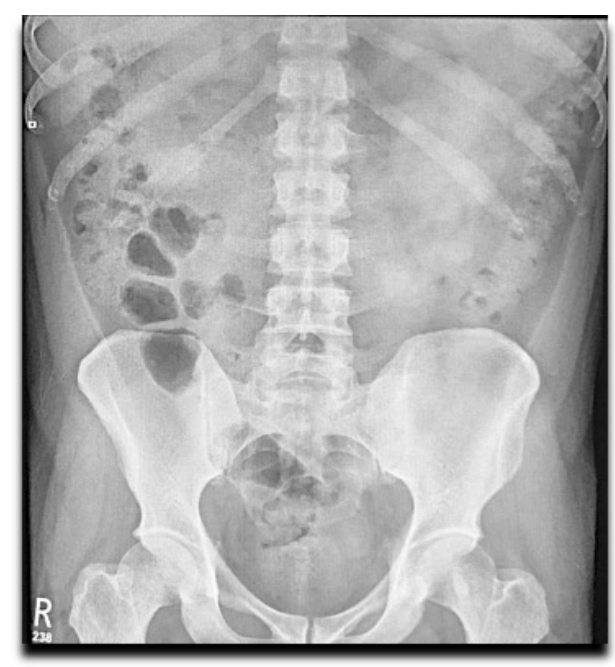

Fig.4: X-ray (kidney, ureter \& bladder) KUB AP view.

studies describing the same for bladder stones. To ensure appropriate evaluation for underlying risk factors, as well as for optimizing the surveillance for bladder stones, further studies are recommended to evaluate the incidence, risk factors, calculus composition and management of such urological presentations. In this case report we have found that patient with large bladder stones can be managed using an endoscopic technique such as percutaneous cystolithotripsy (PCCL) which is a good alternative to the open cystolithotomy.

Contributors: AEO, AhA: manuscript writing and patient management; HA, AlA: manuscript editing and patient management; $\mathrm{AbA}, \mathrm{AJ}$ : critical inputs into the manuscript and patient management. AEO will act as a study guarantor. All authors approved the final version of this manuscript and are responsible for all aspects of the study.

Funding: None; Competing interests: None stated.

\section{References}

1. Schwartz BF, Stoller ML. The vesical calculus. Urologic Clinics of North America. 2000;27(2):333-346.

2. Ahmadnia H, Kamalati A, Younesi M, Imani MM, Moradi M, Esmaeili M. Percutaneous treatment of bladder stones in children: 10 years experience, Is blind access safe? Pediatric Surgery International. 2013;29(7):725-728.

3. Agrawal R, Taha K, Poudyal A, Vidal P, Bhattacharjee P. Giant bladder stone in association with severe kidney injury. Oxford Medical Case Reports. 2019;2019(7):omz063. 
4. Hammad FT, Kaya M, Kazim E. Bladder calculi: Did the clinical picture change? Urology. 2006;67(6):11541158.

5. Torricelli FC, Mazzucchi E, Danilovic A, Coelho RF, Srougi M. Surgical management of bladder stones: literature review. Revista do Colégio Brasileiro de Cirurgiões. 2013;40(3):227-233.
6. Liu G, Deng Y, Zhang S, Zhao X, Yao D, Zhang Q, et al. Minimally invasive percutaneous suprapubic cystolithotripsy: an effective treatment for bladder stones with urethral strictures. Int J Clin Exp Med. 2016;9(10):19907-19912. 